\title{
Шеин В.В., аспирант
}

Академия архитектуры и искусств Южного Федерального Университета

\section{НАПРАВЛЕНИЯ ТРАНСФОРМАЦИИ РЕКОНСТРУИРУЕМЫХ ПРОМЫШЛЕННЫХ ЗДАНИЙ НА ПРИМЕРЕ ОБЬЕКТОВ ЮЖНОГО ФЕДЕРАЛЬНОГО ОКРУГА}

В настоящее время наблюдается рост объемов отечественного промышленного производства, что обусловлено влиянием таких факторов, как политика импортозамещения в стране, государственная програмиа поддержки предпринимательства и малого бизнеса и т.п. Соответственно растет потребность в гибкой, рационально сформированной, высокотехнологичной, экологически чистой, энергоэффективной и эстетически ценной производственной среде. На территории Южного федерального округа расположено более 100 крупных промышленных предприятий, возведенных в советский период, которые имеют потенииал для реконструкиии и дальнейшего использования в производственных иелях, т.е. как пассивные основные фонды промышленного предприятия. В статье приведены результаты исследования промышленных зданий советского периода как архитектурной оболочки современных производственных процессов. Описаны схемы техпроцессов, применяемых в различных отраслях производства. Выделено 4 возможных направления трансформации производственного пространства. Сделан вывод об отсутствии взаимосвязи между схемой техпроцесса и типом производственного здания.

Ключевые слова: промышленные здания, реконструкиия промышленных зданий, промышленная архитектура.

Введение. Южный федеральный округ как один из стратегически важных промышленных регионов, представляет ценность для исследования «закономерностей поведения» промышленных предприятий советского периода. Так или иначе, согласно правительственным документам Федерального и регионального уровня, промышленный сектор экономики является одним из наиболее перспективных. Так, при стратегическом планировании с учетом импортозамещения, в 2018 г. проведены статистические исследования, в ходе выявлены характерные особенности динамики развития различных отраслей промышленности на территории южного региона. При этом вырисовывается следующая картина отрасли с наибольшим оборотом средств - это металлообработка и пищевая промышленность, включающие в себя все отраслевые подуровни (выпуск продукции отрасли приборостроения вырос с 2014 по 2018 гг. в 2,3 раза). Под растущие объемы производства необходима современная производственно-технологическая база, в том числе активных основных фондов и пассивных основных фондов предприятия, иначе говоря, элементов, непосредственно участвующих в процессе производства (машины, механизмы, оборудование и пр.) и тех, что осуществляют вспомогательный функционал (линейные объекты (сети, коммуникации, транспортные пути и пр.). Таким образом, возрастает необходимость в комплексном обновлении архитектурной оболочки производственного процесса.

Методология. Методологической основой работы является системно-структурный подход, позволяющий рассмотреть во взаимосвязи основные особенности объекта исследования. Помимо него на всех этапах работы использовались такие методы исследования как графоаналитический, сравнительный анализы графических и текстовых материалов, полученных из литературных источников; натурная фотофиксация и ее визуальный анализ, классификация на основе индуктивных приемов анализа различных взаимосвязанных аспектов работы производственного объекта, таких, как тип производства, тип здания, градостроительное размещение, особенности генерального плана, конструктивная система, направление техпроцесса и пр., анализ влияния технологических и отраслевых особенностей искомого производства на возможность и специфику реконструкции и дальнейшего использования здания в производственных целях.

Основная часть. Отечественный и зарубежный опыт реконструкции промышленных объектов представляет собой 2 принципиально различных подхода. Если в отечественной практике реконструкция промышленного здания с сохранением первоначальной функции считается наиболее рациональным методом изменения производственной среды под обновленный техпроцесс, то в зарубежной - наоборот, старые промышенные здания преимущественно подвергают реновации, т.е. изменению (восстановлению) с изменением первоначальной производственной функции на принципиально иную, например, жилую или общественную. Так или иначе, в современных российских реалиях рационально использовать отечественный подход в силу его экономичности, 
обусловленной наличием ряда производственных объектов, возведенных в советский период и в достаточной степени сохранивших свои конструктивные и прочие эксплуатационные параметры. Обследованные объекты, как правило, представляют собой промышленные здания пролетного типа, преимущественно с железобетонным, реже - смешанным каркасом. Тип транспортировки грузов внутри здания как рельсовый, так и безрельсовый, от чего напрямую зависит высота цехового пространства. Такие здания являлись архитектурной оболочной производственных процессов отрасли машиностроения, т.е. в основном это сборочные, инструментальные цеха [1]. Однако также часто встречаются полифункциональные цеха, иначе говоря, блокированные цеха, соединяющие под единым объемом различные функциональные участки. Неэксплуатируемые блокированные цеха чаще всего встречаются в структуре крупных промышленных предприятий отрасли машиностроения, таких как Ростсельмаш, Зерноградгидроагрегат и др. Предприятия отрасли машиностроения как правило используют т.н. горизонтальные схемы техпроцесса. Т.е. процесс производства осуществляется по горизонтальной цепочке (рис. 1)

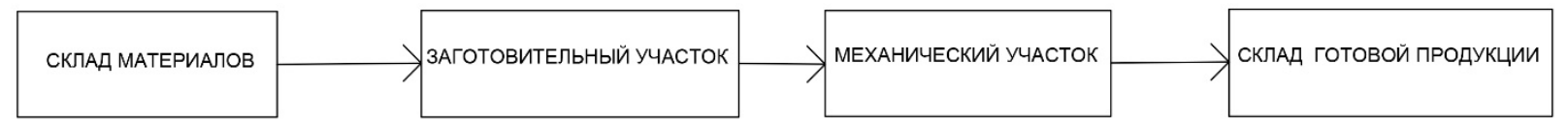

Рис. 1. Горизонтальный техпроцесс

Крупные предприятия приборостроения, такие как КВАНТ также на сегодняшний момент располагаются на небольшой части своих изначальных производственных пространств. Здания отрасли приборостроения преимущественно многоэтажные, ячейковые или, реже - смешанные, соединенные с экспериментальными и сборочными цехами путем организации галерей, прокалывающих последовательно каждый участок технологической цепи. Реконструкция в та- ких зданиях представляет собой техническое перевооружение в совокупности с косметическим ремонтом, облицовкой фасадов вентсистемами [2]. Технологические схемы применяются, как правило, смешанные (рис. 2) либо вертикальные (рис. 3) в силу, часто, мелкоразмерной продукции и специфики применяемых в производстве Машин и Механизмов, а также усложненных требований к среде отдельных технологических этапов производства.

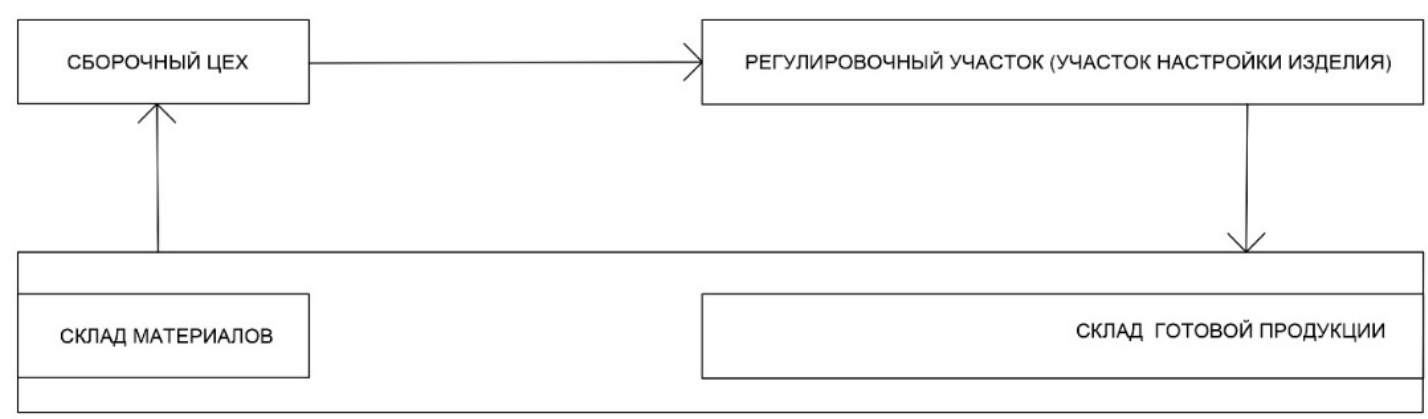

Рис. 2. Смешанный техпроцесс

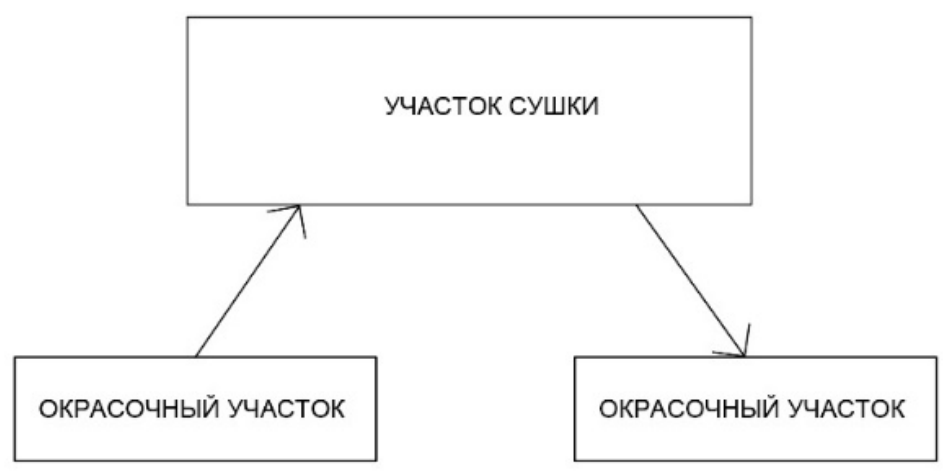

Рис. 3. Вертикальный техпроцесс 
Так или иначе, на настоящем этапе, предприятия различных отраслей промышленности могут быть размещены в зальных, ячейковых и пролетных зданиях путем их коренной реконструкции, проработке объемов по высоте, обеспечения гибкости планировки и мобильности строительных работ при возведении и реконструкции здания благодаря использованию сборно-разборных железобетонных конструкций, а также более легких конструкций из металла, что обуславливается общей тенденцией унификации архитектурной оболочки производственного процесса, разумеется, при наличии благоприятных градостроительных, природно-климатических и пр. условий в соответствии с классом вредности производства.
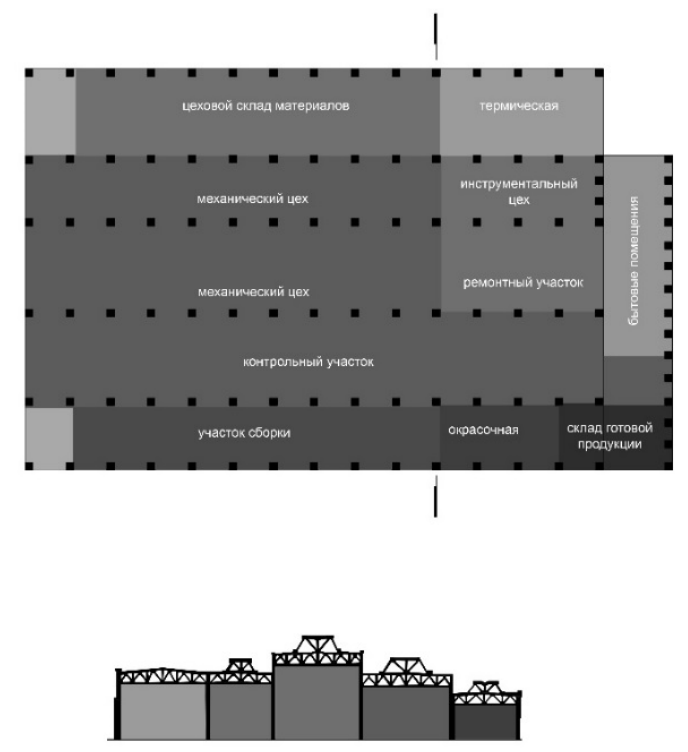

Рис. 4. Функциональная схема размещения различных типов производства в структуре одного архитектурного объема

2. Ориентация при реконструкции на первоначальную производственную направленность с рационализацией использования производственного под новые технологические схемы и измененные габариты оборудования. (рис. 5).

3. В связи с усиленным развитием госпрограмм стратегического планирования с учетом импортозамещения, генерального обновления производственно-технологической базы в стране и ЮФО как одном из развитых, экономически диверсификационных регионов, промышленное производство, так или иначе переориентируется в зависимости от перспективных курсов развития той или иной отрасли.

Так. (уровень капиталооборота частных производственных компаний в ЮФО вырос с 2014 по 2018 гг. на 44,6 \%). По данным росстата

Высокие темпы роста демонстрирует химическая промышленность: выпуск минеральных удобрений вырос в июне на $16,9 \%$, аммиака - на $10,7 \%$, пластмасс в первичных формах - на 15,7
Таким образом, возможны следующие направления трансформации реконструируемых промышленных зданий:

1. Реконструируемый объект полностью унифицирован, не подчиняется конкретной технологической схеме, поскольку может быть адаптирован практически к любой, при наличии соответствия требованиям (градостроительным [4], природно-экологическим, специальным и пр.). Таким образом, настоящий подход предполагает формирование универсальной оболочки производственного процесса, иначе говоря, бывший инструментальный цех может вместить в себя предприятие пищевой промышленности и т.п. (рис. 4).
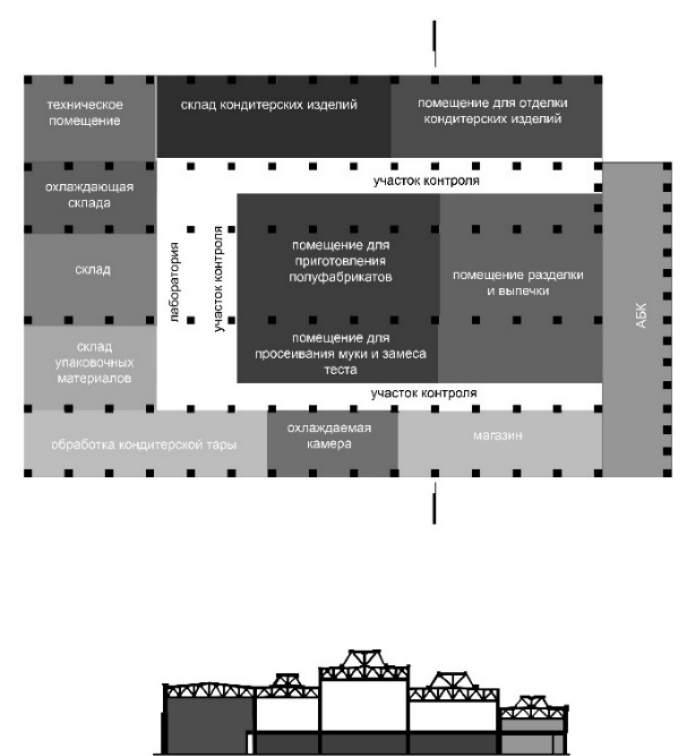

\%. В машиностроении в июне увеличилось производство потребительских товаров длительного пользования - компьютеров (в 2,3 раза), стиральных машин $(+23,4 \%), \quad$ холодильников (+8,5 \%). Вырос выпуск легковых $(+16,9 \%)$ и грузовых (+9,4\%) автомобилей, грузовых вагонов (в 2,3 раза).

Доминирующие виды деятельности, обеспечивающие $75 \%$ отгруженной продукции собственного производства обрабатывающей промышленности, включает в себя «Производство пищевых продуктов, включая напитки, и табака», «Металлургическое производство и производство готовых металлических изделий», «Производство транспортных средств и оборудования», «Производство кокса и нефтепродуктов» и «Производство машин и оборудования». Таким образом, в силу диверсификционного характера экономики региона, рационально использовать принцип гибкой планировки производственных 
зданий для обеспечения непрерывности производственного процесса в период перепрофилирования, расширения или уплотнения того или иного производства, расположенного в конкретной архитектурной оболочке [3, 9].

4. В последние годы активно развивается наукоемкая промышленность, в том числе на территории Южного федерального округа появляются объекты инновационного производства [5], развивающиеся как в пределах кластерных градостроительных образований, так и в пределах одного здания. Необходимо отметить, что, как правило, здания используются существующие, реконструированные $[5,7,8]$. (рис. 6), однако их реконструкция чаще всего представляет собой разработку внутреннего пространства путем организации внутри здания различных функциональных зон, таких, как лаборатории, административно-бытовая часть, экспериментальная и производственная зоны. Однако наукоемкие технологии, в частности - нано, предъявляют к своей оболочке достаточно жесткие требования, такие, как герметичность производственной среды, т.е. защита от дневного света, пыли, электромагнитного излучения, внешнего и внутреннего радиационного излучения и пр., что предполагает формирование в структуре существующего промышленного здания некой герметичной капсулы, обрамленной защитным коридором, с совершенно иной системой организации людских потоков. К сожалению, на настоящий момент примеры такой реконструкции существующего производственного объема отсутствуют, в связи с чем ведется разработка этого вопроса в пределах диссертационного исследования по теме реконструкции существующих промышленных объектов.
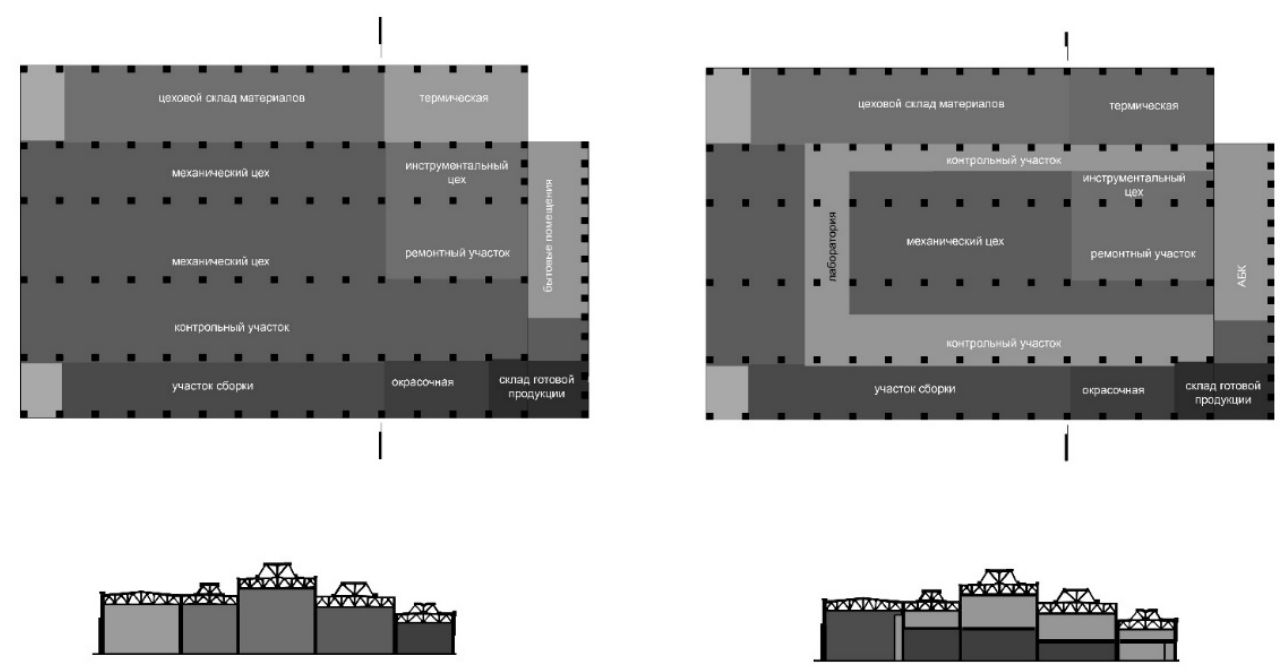

Рис. 5. Функциональная схема трансформации реконструируемого здания в рамках одной отрасли (машиностроение)
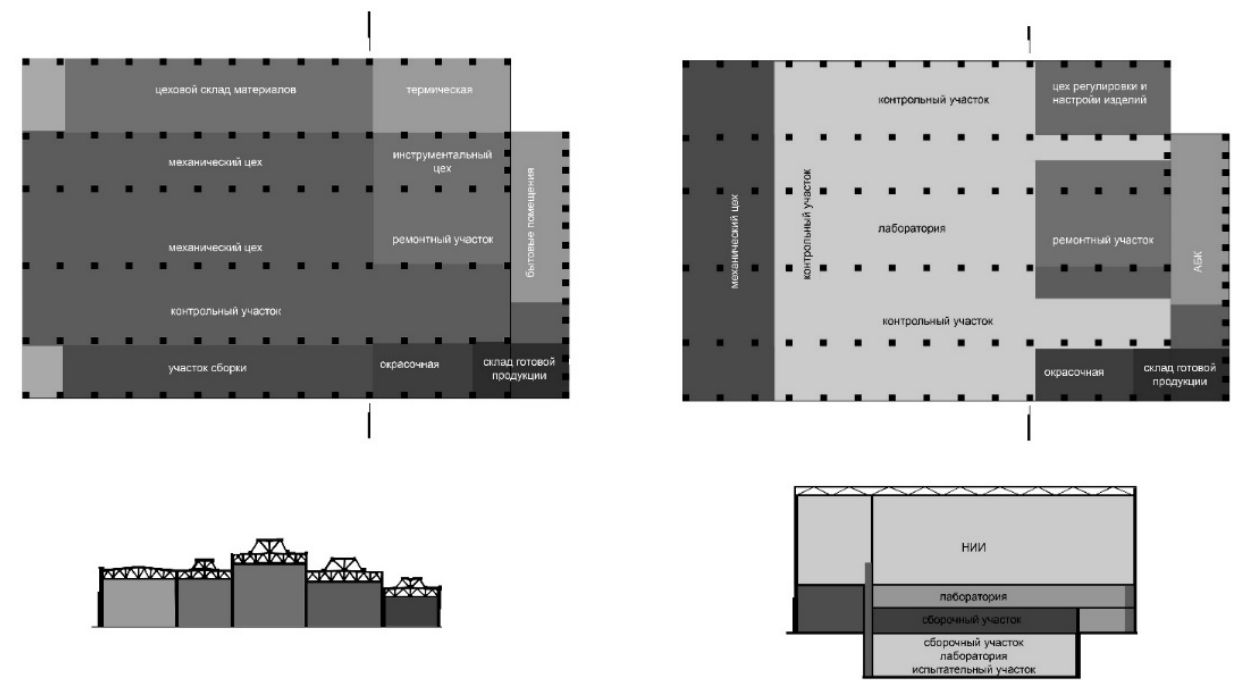

Рис. 6. Функциональная схема трансформации реконструируемого здания в объем, вмещающий наукоемкое производство 
Выводы. В ходе исследования было выявлено отсутствие зависимости типа здания от современных технологических процессов промышленного производства в силу возможности трансформации существующего здания под любой, проходящий внутри техпроцесс при помощи применения конструктивных систем, позволяющих организовать гибкое пространство для производственных и вспомогательных целей. Описано 4 направления возможной трансформации реконструируемых промышленных зданий советского периода: первое направление представляет собой использование существующего объема под техпроцесс любого из описанных типов (горизонтальный, вертикальный, смешанный), под любое производство; второе направление тяготеет к преемственности искомой отраслевой направленности, с учетом которой здание было спроектировано и возведено; третье направление подразумевает возможность диверсификации производственного пространства под изменения параметров перспективности конкретной отрасли с комфортной трансформацией производственной среды; четвертое направление представляет собой, безусловно, коренное изменение среды в силу специфических требований к пространству наукоемкого производства, например таких, как герметизация производственного и испытательного пространства при работе с нано-частицами, - это означает разработку подземного пространства существующего здания, решение здания по высоте, организацию специальных функциональных зон, а также применение прочих объемно-планировочных приемов по созданию производственной среды.

\section{БИБЛИОГРАФИЧЕСКИЙ СПИСОК}

1. Дегтярь А.Н., Серых И.Р., Панченко Л.А., Чернышева Е.В. Остаточный ресурс конструкций зданий и сооружений // Вестник БГТУ им. В.Г. Шухова. 2017. №10. С. 94-98.

2. Сулейманова Л.А. Высококачественные энергосберегающие и конкурентоспособные строительные материалы, изделия и конструкции // Вестник БГТУ им. В.Г. Шухова. 2017. №1. С. 917.

3. Shi S., et al., Deposition velocity of fine and ultrafine particles onto manikin surfaces in indoor environment of different facial air speeds. Building and Environment. 2014. Vol. 81. Pp. 388-395.

4. Перькова М.В. Методика выявления и разрешения градостроительных противоречий на региональном уровне // Вестник БГТУ им. В.Г. Шухова. 2018. №4. С. 62-72.

5. Мальцева Е.В., Никифоров Ю.А. Роль инновационных разработок в современной промышленной архитектуре // Вестник БГТУ им. В.Г. Шухова. 2018. №1. С. 66-71.

6. Shi Pu. Knowledge based building facade reconstruction from laser point clouds and images. Publications on Geodesy 75 NCG Nederlandse Commissie voor Geodesie Netherlands Geodetic Commission. Delft, March 2010, 133 p.

7. Branko P. slavković. application of the double skin façade in rehabilitation of the industrial buildings in serbia. State University of Novi Pazar, Novi Pazar, Serbia, 12p.

8. Jones E. Industrial architecture in Britain: 1750-1939 // J. Edgar. NY: Facts on File. 1985. Vol. $239 \mathrm{p}$.

9. Бергер М.П. Устойчивость поврежденной большепролетной фермы в составе пространственного каркаса // Вестник БГТУ им. В.Г. Шухова. 2018. №2. С. 27-34.

10. Baldinell G., Double skin façades for warm climate regions: Analysis of a solution with anintegrated movable shading system // Building and Environment. 2009. Vol. 44. Pp. 1107-1118.

Информачия об авторах

Шеин Валерия Вячеславовна, аспирант.

E-mail: va_in@list.ru

Академия архитектуры и искусств Южного федерального университета.

Россия, 344082, Ростов-на-Дону, пр. Буденновский, д. 39.

Поступила в июне 2018 г.

(C) Шеин В.В., 2018

\section{Shein V.V. \\ DIRECTIONS OF RECONSTRUCTED INDUSTRIAL BUILDINGS TRANSFORMATION ON THE EXAMPLE OF SOUTHERN REGION OBJECTS}

Growing level of domestic industry is observed at contemporary moment because of a few reasons, such as federal politics of import, substitution and support of small business structures etc. Therefore, the necessity of industrial spaces are formed according to flexibility requirements. Manufacturability, energy efficiency and aesthetics are growing accordingly. There are more than 100 industrial objects situated on Southern region. They were built in Soviet period. Such buildings are potentially attractive to be systematically reconstructed 
and further used as spaces for modern industrial process. The article consists of results of researching existing industrial buildings of soviet period as the shell of contemporary technological processes. Different schemes of technological processes for different types of industry were described at the article. 4 ways of transformation of industrial spaces were detected at the course of the research. It was concluded that there is no connection between direction of technological process and type of building.

Keywords: industrial buildings, reconstruction of industrial buildings, industrial architecture.

\section{REFERENCES}

1. Degtyar A.N., Serykh I.R., Panchenko L.A., Chernysheva E.V. Residual resource of construction of buildings and constructions. Bulletin of BSTU named after V.G. Shukhov, 2017, no. 10, pp. 94-98.

2. Suleymanova L.A. High qualitied energysaving and competitive building materials, products and constructions. Bulletin of BSTU named after V.G. Shukhov, 2017, no. 1, pp. 9-17.

3. Shi S., et al., Deposition velocity of fine and ultrafine particles onto manikin surfaces in indoor environment of different facial air speeds. Building and Environment, 2014, vol. 81, pp. 388-395.

4. Perkova M.V. Methodic of detection and decition of urban contradictions on regional level. Bulletin of BSTU named after V.G. Shukhov, 2018, no. 4, pp. 62-72.

5. Maltseva E.V., Nikiforov The role of innovative elaborations at contamporary industrial architecture. Bulletin of BSTU named after V.G.
Shukhov, 2018, no. 1, pp. 66-71.

6. Shi Pu. Knowledge based building facade reconstruction from laser point clouds and images. Publications on Geodesy 75 NCG Nederlandse Commissie voor Geodesie Netherlands Geodetic Commission. Delft, March 2010, 133 p.

7. Branko P. slavković. application of the double skin façade in rehabilitation of the industrial buildings in serbia. State University of Novi Pazar, Novi Pazar, Serbia, 12 p.

8. Jones E. Industrial architecture in Britain: 1750-1939. J. Edgar. NY: Facts on File, 1985. 239 p.

9. Berger M.P. Sustainability of damaged long span farm as part of space skeleton. Bulletin of BSTU named after V.G. Shukhov, 2018, no. 2, pp. 27-34.

10. Baldinelli G., Double skin façades for warm climate regions: Analysis of a solution with anintegrated movable shading system. Building and Environment, 2009, vol. 44, pp. 1107-1118.

\section{Information about the author}

Valeriya V. Shein, Postgraduate student.

E-mail: va_in@list.ru

Southern Federal University, Academy of architecture \& arts.

Russia, 344082, Rostov-on-Don st. Budennovsky, 39.

Received in June 2018

\section{Для цитирования:}

Шеин В.В. Направления трансформации реконструируемых промышленных зданий на примере объектов Южного федерального округа // Вестник БГТУ им. В.Г. Шухова. 2018. №9. С. 62-67. DOI: $10.12737 /$ article_5bab4a1d21c144.34787598

\section{For citation:}

Shein V.V. Directions of reconstructed industrial buildings transformation on the example of southern region objects. Bulletin of BSTU named after V.G. Shukhov, 2018, no. 9, pp. 62-67. DOI: $10.12737 /$ article_5bab4a1d21c144.34787598 